\title{
Identification of Brain Tumour in Histopathological Images using Neural Networks
}

\author{
P V V S Srinivas, Ch U V Subhash, B Haswanth, Ch Lolesh,
}

\begin{abstract}
Brain tumour is a rare explosion of cells present in brain and it is of two types such as benign and malignant. Mainly tumours occur anywhere in brain irrespective of its size, variance and structure. Without using MRI scan this dangerous brain tumour cannot be identified. The present effective way for brainstorming MRI pictures present in paper. Genuine datasets by dissimilar tumour figures, magnitudes, localities and inner surface are reserved. We take out the significant data (a tumour) from efficient separation utilizing improved convolution neural network (CNN) wherever Elman network is elaborated. So for this reason we determine the improved CNN built method by using MATLAB simulation.
\end{abstract}

Keywords: Brain tumour, Gentle, Malicious, CNN and MRI

\section{INTRODUCTION}

Evolving past or harmed fresh cells result in growing and controlling. To completely understand the causes, tumour cells are duplicated irrepressibly. It will be a surprising improvement in the brain. Brain tumours might be destructive or exposed. A considerate brain tumour need moderate development and need exceptional limit Furthermore might infrequently spread regardless of cell will be not viral. Favourable tumours will make an aggregation danger finishing though placed over magic territory.

A threatening brain tumour grows rapidly, spreads by unpredictable boundary and contiguous rarely spread. Those brain tumours don't apply of the meaning for disease similarly as it doesn't range separate in the brain.

Human brain can't grow of the developing impostor of the brain. Subsequently it brings about those structuring of tumour and unusual brain tissues. Certain brain tumours bring about a obstructive for cerebrospinal fluid (CSF) that permits over that brain and the vertebral line. This boundary expands infrared weight Also might expansion hydroxides. Exactly brain tumours result in swelling (oedema). Quantity, pressure, Furthermore aggravation need aid that climax bringing about those indications of that brain tumour.

Revised Manuscript Received on October 22, 2019.

P V V S Srinivas, Asst. Professor, Dept. Of CSE, KL Deemed to be University, Vijayawada, Andhra Pradesh.

Ch U V Subhash, UG Student, Dept. Of CSE, KL Deemed to be University, Vijayawada, Andhra Pradesh.

B Haswanth, UG Student, Dept. Of CSE, KL Deemed to be University, Vijayawada, Andhra Pradesh.

Ch Lolesh, UG Student, Dept. Of CSE, KL Deemed to be University, Vijayawada, Andhra Pradesh.
Mind tumours can result in harm by block of the tissues alternately through expanding intra-cranial weight. Different restrictions such as measure of the tumour its sort and also the area in the brain define the situation and symptoms

Indiscriminate indications contain:

- Sickness in early morning

- Passiveness

- Dilemma, dazed sensation and walking difficulty

- Crates problem in speech

- Chromatic problems comparable infrequent activities of the eye

- Restlessness, annoyance, sickness and vomiting, inactive reactions.

The brain tumours o numerous brain areas Particular /Restricted indications comprise: Self-destructive considerations and conduct and enthusiastic changes; powerless judgment, disheartening or inhibition, Paralysis, diminished mental capability and recollection misfortune. Powerless speaking, Text, representation or identifying problems, Unrecognized, interior issue and eyelid deterioration, Smeared vision, illusions. Brief nerve tumours can foundation challenge over dialect cognizance. Memory deterioration is more Forceful self-destructive considerations and conduct.

\section{IMAGE SEGMENTATION}

Image segmentation embraces its intending by the parcelling about whatever picture that is advanced under a significant number segments ordinarily known as by pixels. The dream from claiming picture division will be rearrangements about picture representational and also dissection will be aggravated simpler. Picture division empowers the area from claiming Questions Furthermore limit such as curves, lines, and so forth. Here the picture is likewise labels every pixel to a picture such-and-such the pixel for same mark imparts certain aspects. Those resultant divided picture comprises of a set for segments which disguise those whole picture. The pixels of a district are comparable for portion aspects which can make power shade or composition. For the most part with respect to picture division as stack on medicinal imaging, those coming about forms following picture division need aid used to make 3Dimensional reconstructions by utilizing insertion calculations for example, such that walking cubes. 
Iii. Existing framework. That statistical region migration (SRM) will be a calculation utilized within that picture area. [1] Those strategy will be used to assess valuations to a territorial interim and to blend that groundwork of the unification basis, bringing about a little rundown. Percentage functional illustrations give a era assembly in the residents, alternately gathering those nearby pixels In light of those shadows tumbling under a specific passage (qualification criterion) to image transforming. The perfect starts for a portion of a pixel Furthermore will be utilized within measurable experimentation to nearby circles (rising request of essential alterations).

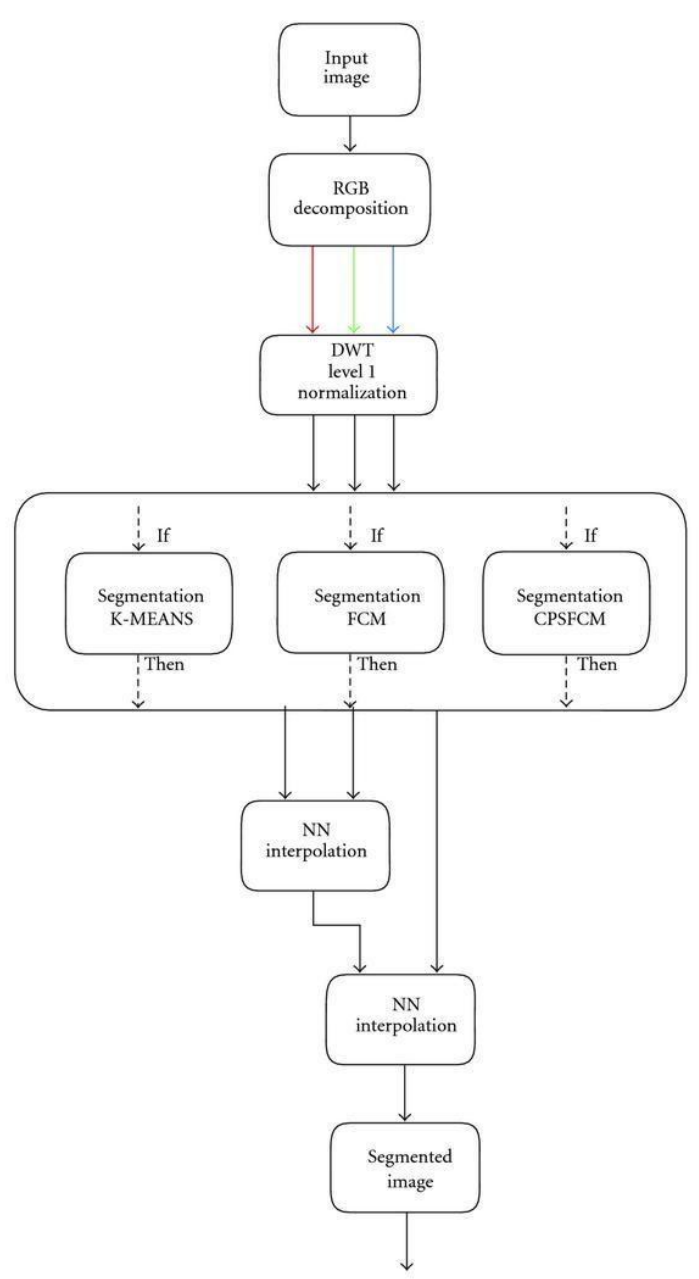

Fig 3 existing system block diagram

\section{PROPOSED SYSTEM}

In this paper, we suggest design and usage of tumour identification starting with cerebrum MR pictures utilizing altered CNN algorithm utilizing Elman system.

This article gives a dependable identification technique that increments $\mathrm{CNN}$-based precision. A convolution neural system (CNN) will be utilized displacing kernel set up of a picture to getting the recuperated plan. Those image transforming systems directing, including transformation of image, removal about structures and adjustment of histogram clear lifestyle to identification and division of the gliomas or tumours those resulting picture might demonstrate will be reasonable and exact as contrasted with current effects.

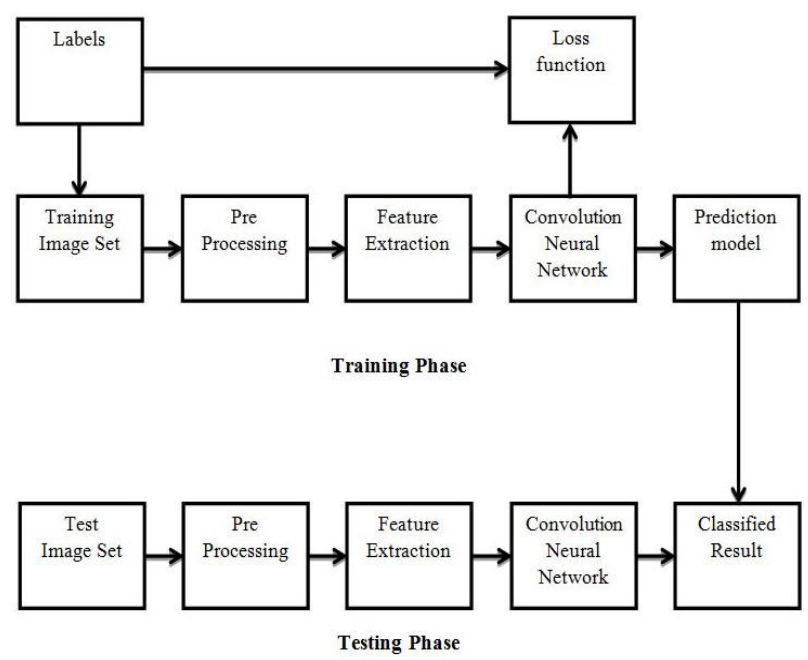

FIG 4. BLOCK DIAGRAM OF PROPOSED METHOD

\section{METHODOLOGY}

A all advanced image transforming framework comprises the accompanying phases on acquire those fancied outcome: [1] picture demonstrating. [2] Picture extension. [3] Characteristic removal. [4] Recognition and identification. Picture Modelling: here it incorporates amount about phases will be as takes after. Perused the enter image: those information picture is stacked utilizing those uigetfile () function, which may be MATLAB inherent purpose, and the picture window may be shown utilizing the im show () work and presentation in the picture window.

RGB with Gray: RGB picture need 3 arrays about red, green and blue, its diligent to eat, with the goal gray is committed Plus its exceptionally of service And viable. Greyscale $=\mathrm{r}+\mathrm{g}$ $+\mathrm{b} / 3$ Gray level, 3 touch picture is $23=8$ gray level (reaching from 0 with 7 ) will be pixel thickness assortments since 0 on 7 in the image. Rgb2gray () work will be utilized. Complexity Normalization: the Contrast of a picture could a chance to be improved utilizing those I'm conform () capacity. That is, that power esteem in the picture is developed. Opposite of the default, it will be known as an alternate plug done. It will be used to move forward that nature of the novel into a film.

Picture expansion: These steps need aid as takes after: histogram Balancing: the Histeq () capacity may be utilized to the histogram comparison of the gray-scale image that utilizes the conform gray size previously, a image.

Characteristic Removal: should get this feature, multi-resolution will be deteriorated. A few determinations are separated, for example, So HH (high-high), hl (high-low), lh (low-high) Furthermore LL (low low). It may be made about 4 separate guidelines similar to vertical direction, level direction, cross heading Furthermore precise course. 
Tip of the tumour need secondary strength, thereabouts it may be fundamental to change those data transfer capacity. Recognition and Identification: the combination of the film is made among that unique picture and the feature concentrated picture.

Convolution neural system $(\mathrm{CNN})$ :. CNNs are escalated consideration ANNs, principally utilized to picture order. (eg alluding of the name they see), grouping for solidarity (image search), Furthermore distinguishing the item to show. There are indistinguishable twin of the Different parts of faces, individuals, road signs, tumours, vertebrae Furthermore visual information Convolution networks perform optical character Recognition (OCR) Also settling on dialect transforming over simple And composed documentation CNN will be utilized for blazing requisitions. That's only the tip of the iceberg recently, Toolbar networks need been specifically utilized for realistic Convolution networks for content Investigation Furthermore map information.

One of the fundamental motivations that this execution of the altered nets (ConvNets or CNNs) in image recognition builds those deep learning abilities of the universe to get excessive upgrades for workstation helped dream which paves manner to self-driven automobiles, robotics, drones, therapeutic diagnostics, and Furthermore dream issue. The picture underneath ordinary convolution neural system.

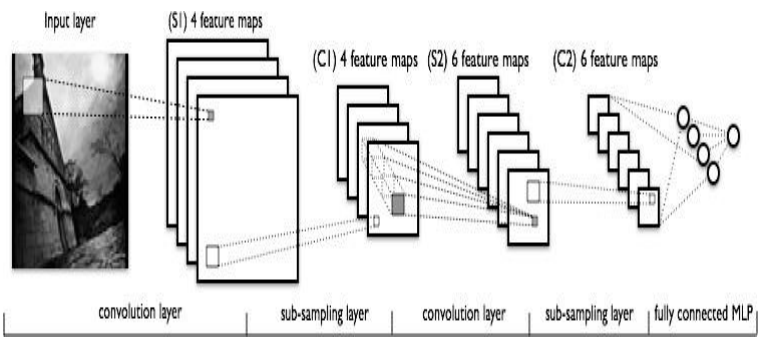

\section{FIG 5. BLOCK DIAGRAM OF CNN}

First information picture scanned for offers.

Practical maps would weight collected, person to every channel we utilization. That huge rectangle is a connection of the downside.

Decrease by execution drawings.

A practical map recently is made on pasquinade screens on the 1st depressed experimented load.

Second resize to fix second capacity maps.

An whole appended layer that categorizes a name yield for a stamp.

Though an ever increasing amount data may be lost, the actuated structures through the reconsidered net ended up additional brief Furthermore a wide margin starting with the visual shapes we perceive and the networks can't gatherings give simple intuitions for profound development.

Elman network: an Elman system is a double layer organize (arranged horizontally concerning illustration $\mathrm{x}, \mathrm{y}$ and $\mathrm{z}$ around a graph) and a "set of units" (examples U). Those standard (enclosed) layer is associated with these biological communities standard with a weight.

Every time, those information and taking in guidelines need aid utilized.

Static-themed joins store those substance of the past values of the last values in units in the connection of the surroundings (they would spreading previously, connection to those taking in system when continuously used). This permits to organize with uphold a sort from claiming state, permitting the performers should perform assignments for example, such that queue-computation past the force of a general multipurpose discernment.

$$
\begin{aligned}
h_{t} & =\sigma_{h}\left(W_{h} x_{t}+U_{h} h_{t-1}+b_{h}\right) \\
y_{t} & =\sigma_{y}\left(W_{y} h_{t}+b_{y}\right)
\end{aligned}
$$

Elman networks bolster ahead networks are and of the again joins linking the plate postponements. For those accessibility about full changing subordinate calculations (fpderiv and btt deriv), Elman organize will be not prescribed but to chronicled Furthermore examination purposes. Attempt a greater amount exact Taking in duration of the time delay (time delay net), layer once again (lea rate), NARX (narx net), Furthermore NAR (nerate) neurological networks.

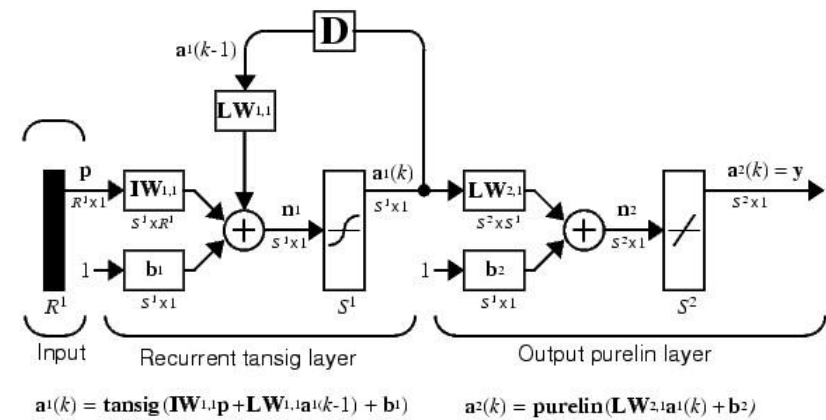

Fig 6. Elman Network layers

Those Elman organize will be a2-layer organize last the initially layer information by initial layer yield. This repeater again connection permits those Elman system with identify Double and make separate types from claiming duration of the time. A two-layer Elman system will be indicated the following. This organize need Dansic neurons over its concealed (repeat) layers, Also its yield layers need immaculate nerves. The blend is exceptional done two layer networks with transactional functions; it might haphazardly align haphazardly for at whatever purpose (with a limited number of crashes). Just those compelling reason for addition neurons of the stowed away layer will be required. Additional convoluted neurons are needed Likewise an intricate issue expanding action. The initial layer need those join go that varies starting with the standard two-layer networks of Elman organize. 
These association postponements the qualities from those past time arrangement utilized within the present period progressive structure. Therefore, both Elman networks, for those same weights Also functions, bring provided for comparative sections in the provided for time form, In spite of their outputs might shift because of fluctuating feedbacks. In light those organize saves majority of the data to future reference, it could gain transitory types and vivified types. Elman organize will be prepared on react and make two sorts about manifestations.

\section{SOFTWARE USED.}

MATLAB will be an instrument which cans computation, visualization also modifying under a client inviting environment, which gives issues Furthermore results to complex issues its provisions include:

a. Math Furthermore computation

b. Algorithm improvement.

c. Sampling

d. Recreation furthermore model.

e. Information analysis

f. Examination and visualization.

g. Science furthermore building Graphics.

h. App Development, including graphical client interface developer.

MATLAB is a crucial grouping with return inside its fundamental information component may be a intuitive system. This permits us should tackle large portions specialized foul computational issues with grid and vector formulas, particularly for a part from claiming time, the point when composing An project to a non-scalar non-interactive dialect such as $\mathrm{C}$, or fortran. The MATLAB form utilized within this task is MATLAB adaptation 2012(MATLAB R2012b).

Provision improvement instruments are such as signal processing, control system, communications, neural network, instrument control, aerospace, fuzzy logic, embedded MATLAB.

\section{CONCLUSION}

Reduced proper sympathetic, in spite of those developing fact innovation done wellbeing supply chains occurs in corporation of methods, those Choice or execution suggestions of innovation reconciliation. Here we executed a productive cerebrum tumour division utilizing changed CNN algorithm directing, including Elman system. Typical CNN based division algorithm provides for handy execution and their precision rate is $82.7133 \%$. Our suggested system provides for preferred exactness rate over the existing technique for $93.9842 \%$ contrasted with $\mathrm{CNN}$ calculation. It might have been compared for Different example enters cerebrum MR Pictures. So we finish up that those altered CNN algorithm directing, including Elman system provides for effective precision rate contrasted with those existing system.

\section{REFERENCES}

1. Pereira, S., Pinto, A., Alves, V., \& Silva, C. A. (2016). Brain tumour segmentation using convolution neural networks in MRI images. IEEE transactions on medical imaging, 35(5), 1240-1251.

2. Nock, R., \& Nielsen, F. (2004). Statistical region merging. IEEE Transactions on pattern analysis and machine intelligence, 26(11), 1452-1458

3. Angulo, J. (2015, October). Statistical Gaussian model of image regions in stochastic watershed segmentation. In International Conference on Networked Geometric Science of Information (pp. 396-405). Springer, Fig13. Neural network training Cham.

4. Tajbakhsh, N., Shin, J. Y., Gurudu, S. R., Hurst, R. T., Kendall, C. B., Gotway, M. B., \& Liang, J. (2016). Convolutional neural Kaus, M. R., Warfield, S. K., Nabavi, A., Black, P. M., Jolesz, F. A., \&Kikinis, R. (2001). Automated segmentation of MR images of brain tumors. Radiology, 218(2), 586-591..

5. Kaus, M. R., Warfield, S. K., Nabavi, A., Black, P. M., Jolesz, F. A., \&Kikinis, R. (2001). Automated segmentation of MR images of brain tumors. Radiology, 218(2), 586-591.

6. Bauer, S., Wiest, R., Nolte, L. P., \& Reyes, M. (2013). A survey of MRI-based medical image analysis for brain tumor studies. Physics in Medicine \& Biology, 58(13), R97.

7. Kalinovsky, A., \&Kovalev, V. (2016). Lung image Segmentation using Fig14. Pattern recognition neural network view deep learning methods and convolutional neural networks.

8. Jiang, F., Grigorev, A., Rho, S., Tian, Z., Fu, Y., Jifara, W., ...\& Liu, S. physics, 44(2), 547-557. 